\title{
Surgical Options for the Treatment of Chronic Pelvic Pain
}

\author{
Vadim V. Morozov ${ }^{1 *}$, Erica C. Dun ${ }^{2}$, Adi Katz ${ }^{3}$ and Kimberly A. Kho ${ }^{4}$ \\ ${ }^{1}$ Obstetrics and Gynecology and Reproductive Sciences, University of Maryland School of Medicine, Baltimore, MD, USA \\ ${ }^{2}$ Obstetrics, Gynecology, and Reproductive Sciences, Yale School of Medicine, New Haven, CT, USA \\ ${ }^{3}$ Obstetrics and Gynecology, Northshore LIJ Health System, Hosftra University, Manhasset, NY, USA \\ ${ }^{4}$ Obstetrics and Gynecology, University of Texas Southwestern Medical Center, Dallas, TX, USA
}

Correspondence to:

Vadim V. Morozov, MD, FACOG, FACS

11 South Paca Street, 4th floor

Baltimore, MD 21201, USA

Tel: 410-328-5964

E-mail: vmorozov@fpi.umaryland.edu

Received: September 25, 2015

Accepted: October 16, 2015

Published: October 20, 2015

Citation: Morozov VV, Dun EC, Katz A, Kho KA. 2015. Surgical Options for the Treatment of Chronic Pelvic Pain. J Neurol Exp Neurosci 1(2): 22-24.

Copyright: (C) 2015 Morozov et al. This is an Open Access article distributed under the terms of the Creative Commons Attribution 4.0 International License (CC-BY) (http://creativecommons. org/licenses/by/4.0/) which permits commercial use, including reproduction, adaptation, and distribution of the article provided the original author and source are credited.

Published by United Scientific Group

\section{Introduction}

Chronic pelvic pain (CPP), particularly pelvic pain among women, remains one of the most controversial and the least understood conditions that practicing gynecologists encounter on a daily basis. It is estimated that this condition affects up to a quarter of all women [1]. The purpose of this article is to describe current surgical management strategies and the success rates of the surgical interventions, in order to help physicians counsel their patients and choose the best surgical option.

Chronic pelvic pain is defined as pain symptoms of at least six months duration that cause functional disability. All pain can be broadly divided into somatic and visceral. These two groups can be further subdivided into nociceptive, inflammatory, neuropathic, psychogenic, mixed, and idiopathic pain [2]. It is of the utmost importance to understand that only nociceptive pelvic pain is potentially amenable to surgical treatment.

\section{Therapeutic Options for Pelvic Pain}

Understanding the anatomical structures involved in generating and perceiving pain is instrumental for developing and implementing therapeutic interventions. The general pathway of pain "sensation" can be grossly described as a sequence of physiologic events: transduction, transmission, modulation, and perception (TTMP) [Figure 1].

Transduction occurs at the site of pain generation - in the case of pelvic pain, in the area generally described as confined to the lower abdomen and pelvis, below the level of the umbilicus, and limited by the gluteal regions as the lower border. In most instances the surgical intervention will take place in this anatomic distribution. Next in the pain pathway is transmission - the process by which the nociceptive stimuli travel through afferent fibers toward the corresponding spinal cord segment. Some surgical interventions can be done at this level of pain-generating pathways, e.g. neurectomies, nerve blocks, nerve injections, and neurolysis. The next echelon of pain propagation is the stage of modulation, which happens in the dorsal roots of the segmental portion of the spinal cord, spinothalamic pathways toward the brain, and the thalamus. This component is characterized by the ascending and descending neuromodulation pathways, such as the diffuse noxious inhibitory control mechanisms (DNIC) [3]. Potentiation or suppression of nociceptive impulses at this level is of crucial importance to the subjective perception of pain. Surgical interventions at this level are rare, yet in some extreme cases of refractory pain (rarely gynecologic pelvic pain), selective surgical stimulation techniques of the spinal cord have been described [4]. 
The last stage of the pain pathway is perception. As demonstrated in [Figure 1], it occurs in the brain cortex and, thus, this topic is beyond the discussion of gynecologic surgical correction of pelvic pain. Our discussion of surgical interventions will primarily focus on the stages of transduction and to a lesser degree on transmission.

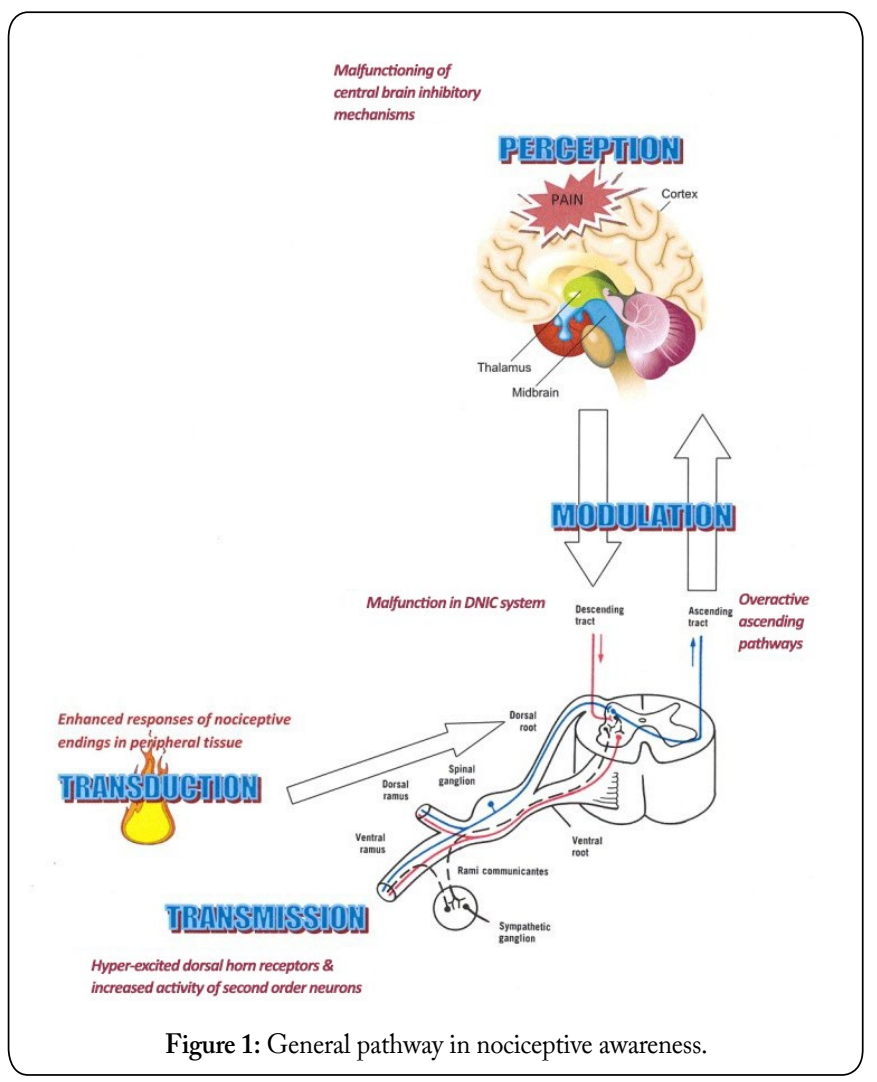

One of the most common nociceptive conditions leading to CPP in the female patient is endometriosis. The pathophysiology of endometriosis, its effects on local surrounding organs and tissues, and the mechanisms by which it causes pain have been studied extensively [2]. As demonstrated by Sutton in 1994 and confirmed by Abbott 10 years later $[5,6]$, both laparoscopic excision of endometriosis and laparoscopic ablation have produced significant reduction in pelvic pain. What is interesting is that the greatest reduction in pain scores after treatment was observed in the highest stages of endometriosis (stages II and III of the American Society for Reproductive Medicine (ASRM) classification), while stage I showed moderate improvement in pain scores [5]. Nevertheless, in a randomized, placebo-controlled trial, laparoscopic excision of endometriosis resulted in significantly more symptomatic relief than laparoscopy alone ( $80 \%$ vs $32 \%$ ) [6].

Pelvic adhesions, also known as pelvic adhesive disease, are another common pathologic condition that can lead to chronic pelvic pain, although the cause and effect between the two are not clearly understood. Keltz et al. demonstrated that lysis of adhesions in the right paracolic region produced a significant reduction of abdomino-pelvic pain in the right and left lower quadrants. Suprapubic and left-sided pain were not affected by right paracolic adhesiolysis [7]. The fundamental concern that surrounds adhesiolysis is that adhesions that had previously been lysed may regenerate. Diamond and Franklin both demonstrated a $70 \%$ rate of adhesion reformation after either laparoscopy or laparotomy in female patients who underwent adhesiolysis, with the ovary having the highest rate of adhesion regeneration, of nearly $80 \%$ [8, 9]. After substantial research into adhesiolysis and its correlation with CPP, the consensus is that preventing the formation of adhesions is an instrumental step towards solving the problem. Meticulous hemostasis, minimizing tissue handling during surgery, the use of non-reactive suture materials, avoiding ischemia, excessive desiccation, and minimal tissue grafting and sponging are among the most common surgical principles that help prevent adhesions.

Pelvic congestion syndrome is a relatively common diagnosis in women with CPP. The condition is characterized by pelvic pain, venous varicosities, and compromised drainage, with resulting venous stasis in the pelvic region. Past management of this condition has relied on both conservative and radical surgical approaches. A conservative surgical approach includes laparoscopic transperitoneal ovarian vein ligation and either clips, suture ligation or coagulation; all have been reported in the past with mixed results $[10,11]$. Due to the limited clinical evidence regarding its efficacy, pelvic vein ligation is currently a controversial treatment of the pelvic congestion syndrome. Most recently, ovarian vessel embolization has been reported, with a significant reduction in symptomatology, but the possibility of migrating embolizing material and the small sample sizes are its main drawbacks [12]. A study that conducted a 12-month follow-up revealed that radical surgical management, such as total hysterectomy and bilateral salpingo-oophorectomy, is successful in treating the pelvic congestion syndrome [13]. Appropriate counseling regarding risks and benefits, including the risks of persistence of pain, should be carefully performed prior to recommending such an extensive surgical intervention.

Pure disorders of pain transmission are relatively rare. The aim of most surgical interventions directed toward this stage is to prevent the transmission of nociceptive impulses to the higher structures of central nervous system. Few surgical procedures in the armament of the gynecologic surgeon exist to affect the transmission of pain impulses. Presacral neurectomy, and nerve transection for mononeuropathy (such as pudendal neuralgia) are the few interventions that have been extensively studied and performed. Laparoscopic utero-sacral nerve ablation (LUNA), which was practiced in the past, has been found ineffective as a robust form of surgical management and thus not recommended for the treatment of CPP [14].

Presacral neurectomy (PSN) is a procedure in which the superior hypogastric nerves and plexus are severed to prevent painful stimuli from reaching the corresponding segments of the spinal cord. This procedure is usually performed laparoscopically. It can be very effective in selected patients with central CPP and dysmenorrhea. PSN was shown to improve pain scores at a 12-month follow-up when performed concurrently with the treatment of endometriosis in all stages of the ASRM classification and in cases of rectovaginal endometriosis [15]. Zulo et al. also showed that rates of dysmenorrhea, dyspareunia, and CPP were significantly lower 
in the PSN group at a 24-month follow-up [16]. The most common factors that led to poor outcomes in the cases of PSN were found to be poor surgical patient selection (patients without a central component of the CPP) and incomplete surgical resection of the presacral plexus [17].

The pudendal nerve, which provides sensory innervation to the clitoral, vulvar, and peri-anal regions, can become injured or physically trapped at several points between its origin at the S2-S4 spinal cord levels and the areas of innervation, leading to debilitating neuralgia and perineal pain. Primary control of this pain, which is usually unilateral, is achieved with local injections and pelvic floor therapy. However, depending on the location of the nerve entrapment, such as in interligamentous space (Alcock canal), the edge of the sacrospinous ligament, or the falciform process of the sacrotuberous ligament, some cases may be refractory [18]. Surgical decompression of the impinged or compressed pudendal nerve has been shown to be effective in $80 \%$ of the cases [19].

\section{Conclusion}

Surgical treatment ofCPP in a female patient is challenging because the pain may not be alleviated with surgery alone. To achieve the greatest benefit and pain relief, patients with CPP require long-term follow-up and a multidisciplinary approach. Understanding the multitude of mechanisms involved in the generation of pain perception, the temporal summation of the pain, and the limits of surgical interventions, should allow the practicing physician to choose the best approach for a particular patient. Even in the best circumstances and with the most favorable surgical outcomes, the complete resolution of CPP may not be an achievable end result. The appropriate counseling of CPP patients is of paramount importance in achieving the balance between the medical and the surgical interventions.

\section{References}

1. Ahangari A. 2014. Prevalence of chronic pelvic pain among women: an updated review. Pain Physician 17(2): 141-147.

2. Howard FM. 2009. Endometriosis and Mechanisms of Pelvic Pain. $J$ Min Invas Gynecol 16(5): 540-550. doi: 10.1016/j.jmig.2009.06.017

3. Le Bars D, Villanueva L, Bouhassira D, Willer JC. 1992. Diffuse noxious inhibitory controls (DNIC) in animals and in man. Patol Fiziol Eksp Ter 4: 55-65. doi: 10.1136/aim.9.2.47

4. Reig E, Abejón D, del Pozo C, Insausti J, Contreras R. 2005. Thermocoagulation of the ganglion impar or ganglion of Walther: description of a modified approach. Preliminary results in chronic, nononcological pain. Pain Pract 5: 103-110. doi: 10.1111/j.15332500.2005.05206.x
5. Sutton CJ, Ewen SP, Whitelaw N, Haines P. 1994. Prospective, randomized, double-blind, controlled trial of laser laparoscopy in the treatment of pelvic pain associated with minimal, mild, and moderate endometriosis. Fertil Steril 62(4): 696-700. doi: 10.1016/00207292(95)99162-A

6. Abbott J, Hawe J, Hunter D, Holmes M, Finn P, et al. 2004. Laparoscopic excision of endometriosis: a randomized, placebo-controlled trial. Fertil Steril 82(4): 878-884. doi: 10.1016/j.fertnstert.2004.03.046

7. Keltz MD, Gera PS, Olive DL. 2006. Prospective randomized trial of right-sided paracolic adhesiolysis for chronic pelvic pain. JSLS 10(4): 443-446.

8. Operative Laparoscopy Study Group Postoperative adhesion development after operative laparoscopy: evaluation at early secondlook procedures. 1991. Fertil Steril 55(4): 700-704.

9. Franklin RR. 1995. Reduction of ovarian adhesions by the use of Interceed. Obstet Gynecol 86(3): 335-340.

10. Mathis BV, Miller JS, Lukens ML, Paluzzi MW. 1995. Pelvic congestion syndrome: a new approach to an unusual problem. Am Surg 61(11): 1016-1018.

11. Takeuchi K, Mochizuki M, Kitagaki S. 1996. Laparoscopic varicocele ligation for pelvic congestion syndrome. Int J Gynaecol Obstet 55(2): 177-178. doi: 10.1016/S0020-7292(96)02733-6

12. Maleux G, Stockx L, Wilms G, Marchal G. 2000. Ovarian vein embolization for the treatment of pelvic congestion syndrome: longterm technical and clinical results. J Vasc Interv Radiol 11(7): 859-864. doi: 10.1016/S1051-0443(07)61801-6

13. Beard RW, Kennedy RG, Gangar KF, Stones RW, Reginal PW, et al. 1991. Bilateral oophorectomy and hysterectomy in the treatment of intractable pelvic pain associated with pelvic congestion. $\mathrm{Br} J$ Obstet Gynecol 98(10): 988-992. doi: 10.1111/j.1471-0528.1991.tb15336.x

14. Daniels J, Gray R, Hills RK, Latthe P, Buckley L, et al. 2009. Laparoscopic uterosacral nerve ablation for alleviating chronic pelvic pain: A randomized controlled trial. JAMA 302(9): 955-961. doi: 10.1001/jama.2009.1268.

15. Zullo F,Palomba S,Zupi E, RussoT,Morelli M, et al.2003. Effectiveness of presacral neurectomy in women with severe dysmenorrhea caused by endometriosis who were treated with laparoscopic conservative surgery: a 1-year prospective randomized double-blind controlled trial. Am J Obstet Gynecol 189(1): 5-10. doi: 10.1067/mob.2003.358

16. Zullo F, Palomba S, Zupi E, Russo T, Morelli M, et al. 2004. Longterm effectiveness of presacral neurectomy for the treatment of severe dysmenorrhea due to endometriosis. J Am Assoc Gynecol Laparosc 11(1): 23-28. doi:10.1016/S1074-3804(05)60005-9

17. Tjaden B, Schlaff WD, Kimball A, Rock, JA. 1990. The efficacy of presacral neurectomy for the relief of midline dysmenorrheal. Obstet Gynecol 76(1): 89-91.

18. Robert R, Prat-Pradal D, Labat JJ, Bensignor M, Raoul S, et al. 1998. Anatomic basis of chronic perineal pain: role of the pudendal nerve. Surg Radiol Anat 20(2): 93-98. doi: 10.1007/BF01628908

19. Dellon AL, Coady D, Harris D. 2015. Pelvic pain of pudendal nerve origin: surgical outcomes and learning curve lessons. J Reconstr Microsurg 31(4): 283-290. doi: 10.1055/s-0034-1396896 